addition, monocyte subset counts were determined, based on CD14 and CD16 expression. To obtain absolute counts, proportions determined by flow cytometry were corrected by the total CD4+ T-cell and monocyte counts. Th1, Th17 and monocyte subsets were determined in 21 GCA patients, 19 PMR patients and 19 healthy controls $(\mathrm{HC})$. Th2 cells were determined in 10 GCA patients, 10 PMR patients and $10 \mathrm{HC}$. All GCA and PMR patients were newly-diagnosed and treatment-naive. $\mathrm{HC}$ were age- and sex-matched and without any immunomodulatory medication.

Results: Both absolute counts and percentages of peripheral Th1 cells, Th17 cells and Th2 cells did not differ between GCA/PMR patients and HC. The monocytosis in GCA and PMR was mainly attributed to an expansion of the classical monocyte subset. Counts of monocyte subsets were not strongly correlated with counts of either Th1 or Th17 cell counts. In GCA patients, the ESR correlated positively with counts of intermediate monocytes $(R=0.63)$, but this was not observed in PMR patients.

Conclusion: Compared to most previous work, we report similar circulating Th1 and Th17 cell counts in HC, but lower counts in treatment-naive GCA patients then previously reported (Table 1). Furthermore, numbers of Th1 and Th17 cells in peripheral blood showed no relationship with monocyte subsets. As our protocol for defining Th1 and Th17 cells appears to be similar to the other studies, we propose differences in patient selection. Alternatively, Th1 and Th17 skewing should be studied at the site of inflammation, since Th1 and Th17 skewing cytokines are all highly expressed by macrophages at the inflammatory site. This study shows the importance of replicating previous research, as key concepts of disease pathology are derived from data on disturbed Th cell distribution.

Table 1. Observed median percentages of circulating Th1 and Th17 cells in GCA patients and age-matched HC. Shown are outcomes from several previous studies as well as the present study. Deng et al, Terrier et al and Saadoun et al found elevated Th1 cell percentages in GCA, whereas Samson et al found them lower. All four previous studies showed elevated Th17 percentages in GCA.

\begin{tabular}{lcccc}
\hline & Th1\% & \multicolumn{3}{c}{ Th17\% } \\
\hline Reference & GCA & HC & GCA & HC \\
\hline Deng et al., Circulation, 2010 & 20 & 12 & 2.2 & 0.4 \\
Terrier et al., Arthritis Rheumatol, 2012 & 22 & 12 & 2.5 & 0.5 \\
Saadoun et al., Arthritis Rheumatol, 2015 & 22 & 17 & 2.5 & 0.6 \\
Samson et al., Arthritis Rheumatol, 2012 & 10 & 14 & 0.7 & 0.3 \\
This study & $\mathbf{1 1}$ & $\mathbf{1 2}$ & $\mathbf{0 . 6}$ & $\mathbf{0 . 5}$ \\
\hline
\end{tabular}

Disclosure of Interests: Yannick van Sleen: None declared, Elisabeth Brouwer Consultant of: Roche (consultancy fee 2017 and 2018 paid to the UMCG), Speakers bureau: Roche (2017 and 2018 paid to the UMCG), Minke G. Huitema: None declared, Wayel Abdulahad: None declared, Maria Sandovici: None declared, Annemieke Boots Consultant of: Grünenthal Gmbh until 2017, Kornelis van der Geest Speakers bureau: Roche (2019)

DOI: 10.1136/annrheumdis-2020-eular.4499

\begin{tabular}{|l|l}
\hline AB0047 & T-CELL IMMUNOGLOBULIN AND MUCIN \\
DOMAIN-CONTAINING PROTEIN 3 EXPRESSION \\
IN REGULATORY T CELLS OF ANKYLOSING \\
SPONDYLITIS PATIENTS AND THE CORRELATION \\
WITH DISEASE ACTIVITY
\end{tabular}

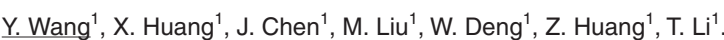

${ }^{1}$ Guangdong Second Provincial Central Hospital, Department of Rheumatology and Immunology, Guangzhou, China

Background: Ankylosing spondylitis (AS) is a chronic inflammatory autoimmune disease. Regulatory T cells have been found in peripheral blood of AS patients. However, there is a controversy regarding the relative number and function of regulatory T cells in AS. T-cell immunoglobulin and mucin domain-containing protein 3 (Tim-3) is a negative immune regulator that participates in immune responses and widely expresses on a variety of immune cells. Many studies have shown that Tim-3 participates in tumors, infections, hematological system diseases and autoimmune diseases (such as systemic lupus erythematosus, rheumatoid arthritis, autoimmune hepatitis, etc). While there has not yet clinical trials with large samples to verify whether or not Tim-3 is involved in the incidence of AS and the relationship between the disease activity of AS and the expression of Tim-3 in regulatory T cells. Objectives: The present study aimed to identify the Tim-3 expression in regulatory T cells in AS patients, and the association between Tim-3 and the disease activity of AS.

Methods: There were 47 patients diagnosed as AS and 51 age- and sexmatched healthy controls (HCs) from Guangdong Second Provincial Central
Hospital enrolled in the study. The clinical information of the AS patients was recorded in detail and the disease activity was calculated. The positive expression rates and medium fuorescence intensity (MFI) of Tim-3 in regulatory $\mathrm{T}$ cells were examined by fow cytometry. Statistical approaches were used to analyze the experimental data of patients and controls.

Results: The Treg cells level was significantly decreased in AS patients compared to the healthy controls $(5.14 \pm 0.27$ vs $4.38 \pm 0.23, P=0.032$, Fig $1 \mathrm{~A})$. Tim-3 expression in regulatory T cells was lower in AS patients than the HCs $(63.29 \pm 2.39$ vs $52.56 \pm 3.49, \mathrm{P}=0.013$, Fig1 $\mathrm{B})$. And Tim-3 MFI in regulatory $T$ cells was significantly decreased too $(1355.04 \pm 171.44$ vs $859.19 \pm 105.11, P=$ 0.016, Fig1 C).

The Treg cells ratio negatively correlated with BASDAI $(r=-0395, p=0.014)$ and BASFI ( $r=-0391, p=0.015)$ in AS patients. However, the Tim-3 expression in the Treg cells has no correlation with diseases activity in patients.
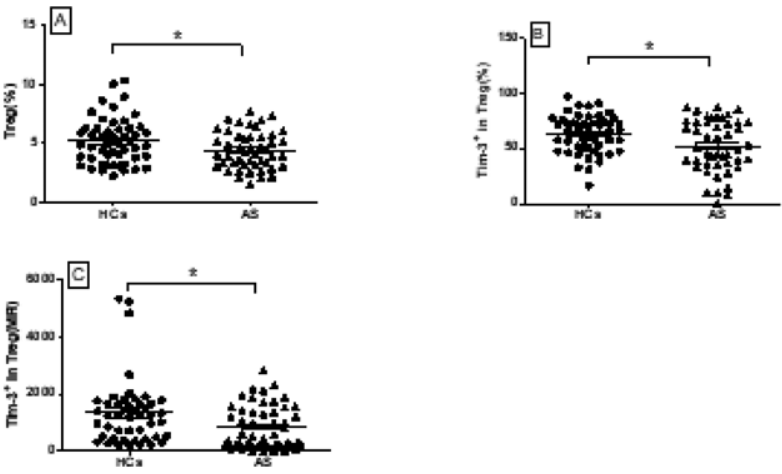

Figurel The Treg cells frequency, Tim-3 expression and MFI in AS

patients compared to the healthy controls.

Conclusion: According to the test results, we could confirm that regulatory $T$ cells participate in the progression of AS, and it has negative relationship with disease activity. Tim-3 can express in Treg, but whether Tim-3 can be used as a potential target for AS treatment in future need further verified.

Disclosure of Interests: None declared

DOI: 10.1136/annrheumdis-2020-eular.5618

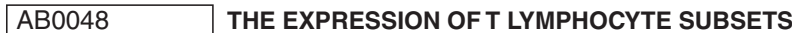 IN PERIPHERAL BLOOD OF PATIENTS WITH AUTOIMMUNE UVEITIS AND THE SHORT-TERM EFFICACY AND SAFETY EVALUATIONS OF LOW-DOSE IL-2 COMBINED THERAPY}

T. Yan ${ }^{1}$, J. Li ${ }^{1} .{ }^{1}$ The Second Hospital of Shanxi Medical University, Taiyuan, China

Background: Autoimmune uveitis is a group of inflammatory diseases that affect the uveal tract such as iris, cilia and choroid. In addition to diabetic retinopathy and age-related macular degeneration, uveitis is one of the main causes of blindness in developed countrie ${ }^{[1]}$. The disease is autoimmune-mediated, and abnormal immune responses are induced by pathogenic antigens such as retinal soluble antigens and retinal interphotoreceptor retinoid-binding protein (IRBP) and autoimmune inflammation is caused by specific cytotoxic effects, immune complex responses and delayed hypersensitivity reactions. It has been found that the disorder of lymphocyte subsets, mainly due to the number and function defects of regulatory T cells (Tregs), may be involved in the development of uveitis. IL-2 is a key cytokine in T cell differentiation. As a new type of immunomodulator, IL-2 has achieved preliminary efficacy in the treatment of systemic lupus erythematosus, ankylosing spondylitis, Sjogren's syndrome and other diseases $^{[2-4]}$, but there is no clinical evidence of IL-2 in the treatment of autoimmune uveitis. The purpose of this study was to investigate the expression of $T$ lymphocytes in patients with autoimmune uveitis and the effect of low dose IL-2 on their immune status.

Objectives: To investigate the expression of peripheral blood lymphocyte in patients with autoimmune uveitis and evaluate the short-term efficacy and safety of low-dose IL-2 combined with methylprednisolone.

Methods: A total of 108 patients with autoimmune uveitis and 93 healthy subjects who visited our hospital from January 2016 to April 2019 were collected.Twenty-three patients were treated with a low dose of IL-2 (50WIU/ day for 5 consecutive days) on the basis of conventional treatment 
(methylprednisolone and/or DMARDs), and the changes in the patients' condition and lymphocyte subsets were observed. The t-test of two independent samples was used when the measurement data conformed to the normal distribution and the variance was homogeneous, and Manil-Whitney rank sum test was used when the measurement data did not conform to the normal distribution.

Results: Among 108 patients, 58 were males and 50 were females, with an average age of $41+14$ years. Compared with the normal control group, total T cells, total B cells, Th cells, Ts cells, Th1 cells, Th17 cells, Th1/Th2, Th17/ Treg in patients with autoimmune uveitis were higher than those in healthy control group $(P<0.05)$, while Th1 cells and Treg cells were lower than those in healthy control group $(P<0.05)$. After IL-2 treatment, the number of Treg cells increased from $21.90 \pm 15.29 / \mathrm{ul}$ to $51.54 \pm 41.86 / \mathrm{ul}(\mathrm{P}<0.05)$, the Th17/ Treg ratio decreased back from $0.44 \pm 0.27$ to $0.33 \pm 0.23(P<0.05)$, and both serum sedimentation rate and CRP decreased compared with before treatment $(P<0.05)$.

Conclusion: Treg cells are involved in the pathogenesis of autoimmune uveitis. Low dose of IL-2 selectively elevates Treg cells, regulates Th17/Treg balance and improves the condition of the disease.

References:

[1] Durrani OM, Meads CA, Murray PIJO. Uveitis: A Potentially Blinding Disease[J]. 2004, 218(4): 223-236.

[2] He J, Zhang X, Wei Y, et al. Low-dose interleukin-2 treatment selectively modulates CD4(+) T cell subsets in patients with systemic lupus erythematosus[J]. 2016, 22(9): 991-993.

[3] An H, Li X, Li F, et al. The absolute counts of peripheral T lymphocyte subsets in patient with ankylosing spondylitis and the effect of low-dose interleukin-2[J]. 2019, 98(15): e15094.

[4] Miao M, Hao Z, Guo Y, et al. Short-term and low-dose IL-2 therapy restores the Th17/Treg balance in the peripheral blood of patients with primary Sjögren's syndrome[J]. 2018: annrheumdis-2018-213036.

Disclosure of Interests: None declared

DOI: 10.1136/annrheumdis-2020-eular.2711

\section{AB0049 IMMUNE DYSFUNCTION IN ANKYLOSING SPONDYLITIS (AS) AND THE POTENTIAL OF TUMOR NECROSIS FACTOR-A (TNF- $\alpha$ ) INHIBITOR ANBAINUO AS AN EFFECTIVE TREATMENT}

M. Yang ${ }^{1}$, Q. Lv ${ }^{1}$, Q. Wei ${ }^{1}$, J. Gu ${ }^{1} .{ }^{1}$ Third Affiliated Hospital of Sun Yat-sen University, Guangzhou, China

Background: Studies into ankylosing spondylitis (AS) and its relationship with immune function are controversial, and the correlation between the efficacy of TNF-a inhibitor and changes in immune function is unclear.

Objectives: We conducted a prospective study of T-cell and B-cell subset distribution and analyzed lymphocyte function in AS patients to further clarify changes to the immune system caused by AS and to explore resistance that could contribute to relapse after treatment.

Methods: A total of 40 immune cells were tested with flow cytometry, and the results of $105 \mathrm{HC}$ (healthy control) subjects, 177 active-stage AS patients, and 23 AS cases before and after 12 weeks of Anbainuo therapy were analyzed.

Results: Compared with the HC group, the proportion of immune cells, such as naïve and central memory CD4+T cells, in AS increased $(p<0.0001)$, but effector memory and terminally differentiated $C D 4+T$ cells were decreased ( $p<0.01$ and 0.0001 , respectively). Naïve, central memory, and effector memory CD8+T cells were increased $(p<0.0001,0.001$, and 0.01 , respectively), but terminally differentiated CD8+T cells were decreased $(p<0.0001)$. Th1 cells (helper T cells-1), Tfh1 cells (follicular helper T cells-1), Tc1 cells (cytotoxic $T$ cells-1), and Tregs (regulatory $T$ cells) were lower $(p<0.01,0.05,0.0001$, and 0.001 , respectively), but Th17 cells, Tfh17 cells, and Tc cells were higher $(p<0.001,0.0001$ and 0.001 , respectively). The proportions of total $B$ cells and class-switched B cells were increased $(p<0.05)$, but non-switched B cells, plasma cells, memory B cells, and immature Bregs (regulatory B cells) were lower ( $p<0.01,0.0001,0.0001$, and 0.0001 , respectively). After Anbainuo therapy, the percentage of Tregs and B10 cells (IL-10-producing regulatory B cells) had increased $(p<0.01$ and 0.05 , respectively), and the increase in Tregs was positively correlated with the decrease in CRP (C-reactive protein) $(r=0.489$, $\mathrm{p}=0.018$ ).

Conclusion: We found that, in terms of both innate and acquired immunity, active-stage AS patients have an immunity imbalance involving multiple types of immune cells, including CD4+T cells, CD8+T cells, Th cells, Tfh cells, Tc cells, Tregs, Bregs, and B cells. Anbainuo can not only help to inhibit disease activity and partial immune function imbalance in AS but can also increase the number of negative regulatory cells in inflammation.

\section{References:}

[1] Long, S., et al., High frequency of circulating follicular helper T cells is correlated with $B$ cell subtypes in patients with ankylosing spondylitis. Exp Ther Med, 2018. 15(5): p. 4578-4586.

[2] An, H., et al., The absolute counts of peripheral $\mathrm{T}$ lymphocyte subsets in patient with ankylosing spondylitis and the effect of low-dose interleukin-2. Medicine (Baltimore), 2019. 98(15): p. e15094.

Acknowledgments: Thanks to Professor Zhinan Yin for his support and assistance with this study

Disclosure of Interests: None declared

DOI: 10.1136/annrheumdis-2020-eular.5119

\section{$\mathrm{AB0050}$}

EXTENDED POLYDIMENSIONAL IMMUNOME CHARACTERISATION (EPIC) PLATFORM AS A TOOL FOR TRANSLATIONAL RESEARCH

J. G. Yeo ${ }^{1,2}$, M. Wasser ${ }^{1}$, P. Kumar ${ }^{1}$, L. Pan ${ }^{1}$, S. L. Poh ${ }^{1}$, F. Ally ${ }^{1}$,

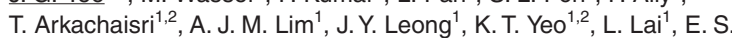
C. Lee ${ }^{2}$, C. Chua ${ }^{1}$, B. Paleja ${ }^{1}$, S. P. Tang ${ }^{3}$, S. K. Ng ${ }^{4}$, A. Y. J. Tan ${ }^{2}$, S. Y. Lee ${ }^{2}$, F. Ginhoux ${ }^{1,5}$, T. P. $\mathrm{Ng}^{6}$, A. Larbi ${ }^{5}$, S. Albani ${ }^{1,2}{ }^{1}$ Translational Immunology Institute, SingHealth Duke-NUS Academic Medical Centre, Singapore, Singapore; ${ }^{2} K K$ Women's and Children's Hospital, Singapore, Singapore; ${ }^{3}$ Selayang Hospital, Paediatric Rheumatology Unit, Department of Pediatrics, Kuala Lumpur, Malaysia; ${ }^{3}$ Selayang Hospital, Paediatric Rheumatology Unit, Department of Pediatrics, Kuala Lumpur, Malaysia; ${ }^{5}$ Agency for Science, Technology and Research, Singapore Immunology Network (SIgN), Singapore, Singapore; ${ }^{6}$ National University of Singapore, Singapore, Singapore

Background: We created a high dimensionality healthy human Immunome atlas by interrogating the peripheral blood mononuclear cells (PBMC) of $>200$ healthy subjects (cord blood to adult) with 63 unique mechanistic and phenotypic markers per cell by mass cytometry (CyTOF). This database is built with an open source, web-based bioinformatics toolkit, enabling its mining and uploading of datasets for comparison with the EPIC healthy database.

Objectives: Here, we demonstrate the platform's ability to identify the immunological differences of mechanistically important cell subsets in the uploaded data in comparison with EPIC.

Methods: CyTOF data from 37 healthy elderly ( $>60$ years old) was uploaded onto the EPIC Discovery tool where down-sampling, normalising and FlowSOM (Flow analysis with Self-Organising Maps) clustering were done with the EPIC database for comparison. Online visualisation outputs include cluster frequency boxplots, correspondence analysis (CA) plot and markers expression heat-map. The CA 2-dimensional plot depicts the global differences in immune cells composition between subjects with proximity between points (subjects) denoting similarity. Kruskal-Wallis test was done to identify age groups differences.

Results: Increasing distances on the CA plot with age were observed with the elderly being farthest from the new-borns. Notably, we observed significant changes in naive $\mathrm{CD} 4^{+} \mathrm{IL} 8^{+} \mathrm{T}$ cells $\left(\mathrm{p}<1 \times 10^{-20}\right)$, memory CD4 $4^{+}$ IL $17 A^{+} T$ cells $\left(p<1 \times 10^{-20}\right)$ and type 2 innate lymphoid cells (ILC2) (Lin CD7 $\mathrm{CD}^{2} 5^{+} \mathrm{CD} 127^{+} \mathrm{CD} 161^{+}, \mathrm{p}<1 \times 10^{-17}$ ) with increasing age. The naive $\mathrm{CD} 4^{+}$ IL8 ${ }^{+} \mathrm{T}$ cells (median: $0.68 \%$, interquartile range: 0.415 to $1.055 \%$ of CD45+ PBMC) and ILC2 $(0.09 \%, 0.065$ to $0.12 \%)$ were lowest and memory IL17A T cells $(0.58 \%, 0.41$ to $0.905 \%)$ highest in the elderly. Significantly, the memory IL17A ${ }^{+} \mathrm{T}$ cells and ILC2 have been implicated in the pathogenesis of auto-immune conditions ${ }^{1,2}$.

Conclusion: With EPIC, we have created an online tool enabling data uploading for comparison to a healthy database, allowing the holistic characterisation of immunological changes in different clinical scenarios. Using it, we were able to identify mechanistically important differences in immune cells composition in a distinct clinical cohort (elderly) compared to the younger ages. Translationally, the EPIC platform can be utilised similarly to catalyse the discovery process in auto-immune diseases interrogated with the EPIC antibody panels.

References:

[1] Fasching P, Stradner M, Graninger W, Dejaco C, Fessler J. Therapeutic Potential of Targeting the Th17/Treg Axis in Autoimmune Disorders. Molecules. 2017 Jan 14;22(1). pii: E134.

[2] Klose CS, Artis D. Innate lymphoid cells as regulators of immunity, inflammation and tissue homeostasis. Nat Immunol. 2016 Jun 21; 17(7): 765-74.

Disclosure of Interests: None declared

DOI: 10.1136/annrheumdis-2020-eular.4068 